\title{
ROLE OF BCG VACCINATION IN THE DIAGNOSIS OF CHILDHOOD TUBERCULOSIS
}

Diagnosis of Tuberculosis in childhood is controversial usually. Diagnostic parameters which are taken under consideration are clinical, routine laboratory and radiological, culture, antigen antibody test. As antigen antibody test are very costly and culture reports take 6 weeks usually these tests are not done frequently. In children, commonly used tests are erythrocyte sedimentation rate (ESR), Montoux test and X-ray chest.

Clinically gradual loss of weight, anorexia, anaemia, lethargy, protein energy malnutrition with anergy to any antigen are the common clinical manifestation. Due to anaemia, ESR is usually high and sometimes due to pyogenic lung infections hilar nodes are often enlarged so it is very difficult to diagnose childhood tuberculosis on the basis of $\mathrm{X}$-ray and ESR. One common antigen antibody test / BCG or Montoux test should be done to diagnose tuberculosis. In $42-50 \%$ cases of protein energy malnutrition cases, Montoux test is negative but BCG test is found to be very sensitive and accurate due to more antigen load. In Kohalpur Teaching Hospital NGMC Nepal, we conducted a study for early diagnosis of tuberculosis by giving $0.05 \mathrm{ml}$
BCG vaccine intradermally and seeing the response on 100 outpatients of pediatrics department, we found $65 \%$ of the patients developed induration more than $10 \mathrm{~mm}$ in size. Patients visiting OPD with either of the following complaints had undergone the test prolong mild, grade fever, loss of weight, anorexia, lymphadenopathy and persistent cough. Patients who had BCG test more then $10 \mathrm{~mm}$ after $48-72$ hours were given anti tubercular drug therapy in recommended doses with three drugs (Pyzina, INH and Rifampicin). Follow up after one montoux those patients who received antitubercular drug on the basis of positive BCG test, started recovering in terms of increase in weight, appertite, activity and subsidence of fever. From our study we conclude that BCG test can be done as a very cheap, reliable, sensitive and easily available for rapid diagnosis of childhood tuberculosis in the community of Nepal.

\section{Dr. Subhajit Banerjee}

Lecturer, Dept. of Pediatrics

Kohalpur Teaching Hospital

NGMC Nepal.

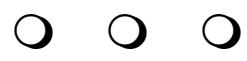

\section{Request to the Readers}

All the readers are encouraged to send the feedback for publication in "Letter to the Editor" section.

JNMA Editorial board welcomes general and specific comments, criticisms which will ultimately guide us to improve the quality of the journal. 